\title{
Computerized Systems Supporting Clinical Decision in Medicine
}

\author{
Aleksander J. Owczarek ${ }^{1}$, Mike Smertka ${ }^{2}$, Przemysław Jędrusik ${ }^{3}$, \\ Anita Gębska-Kuczerowska ${ }^{4}$, Jerzy Chudek ${ }^{5}$, Romuald Wojnicz ${ }^{6}$
}

1 Department of Statistics, Department of Instrumental Analysis, School of Pharmacy with the Division of Laboratory Medicine in Sosnowiec, Medical University of Silesia in Katowice, Poland

2 Pathophysiology Unit, Department of Pathophysiology, School of Medicine in Katowice, Medical University of Silesia in Katowice, Poland

3 Department of Computer Biomedical Systems, Institute of Computer Science, University of Silesia, Poland

4 National Institute of Public Health, National Institute of Hygiene in Warsaw, Poland

5 Department of Internal Medicine and Oncological Chemotherapy, Medical Faculty in Katowice, Medical University of Silesia in Katowice, Poland

${ }^{6}$ Department of Histology and Embryology, School of Medicine with the Division of Dentistry in Zabrze, Medical University of Silesia in Katowice, Poland

\begin{abstract}
Statistics is the science of collection, summarizing, presentation and interpretation of data. Moreover, it yields methods used in the verification of research hypotheses. The presence of a statistician in a research group remarkably improves both the quality of design and research and the optimization of financial resources. Moreover, the involvement of a statistician in a research team helps the physician to effectively utilize the time and energy spent on diagnosing, which is an important aspect in view of limited healthcare resources. Precise, properly designed and implemented Computerized Clinical Decision Support Systems certainly lead to the improvement of healthcare and the quality of medical services, which increases patient satisfaction and reduces financial burdens on healthcare systems.
\end{abstract}

\section{Introduction}

Statistics is the science of collection, summarizing, presentation and interpretation of data. Additionally, it yields methods used in the verification of the research hypotheses. In medicine, statistics is mainly used in: medical practice (so-called hospital statistics), management of medical services (e.g. epidemiology of diseases), and clinical medicine (e.g. assessment of the accuracy of measurements and the quality of diagnostic tests) 
(Szydło, 2005). Nowadays, for medical practitioners, a biostatistician is a partner who ensures the correct design of experiments, analyses, result interpretation, and hypothesis verification. Furthermore, they make a contribution to the development and performance of new diagnostic methods, which enable an improvement of the quality of medical care. Participation of a statistician in a research group has a significant impact not only on the quality of planning and implementation, but also on the optimization of the related financial expenditures. Moreover, time and energy are saved, which is important for healthcare systems. Another aspect of this cooperation is the opportunity to create new methods and solutions to problems arising in clinical practice (Dobbson, 1983; Szydło, 2005). The best example of such a collaboration is the so-called Computerized Clinical Decision Support Systems - CCDSS (Garg et al., 2005; Hughes, 2009; Sim et al., 2001).

Clinical Decision Support Systems, in which various types of inference methodology are employed, have been widely and extensively used in many fields of medicine (Berlin et al., 2006; Eom et al., 2008; Kawamoto et al., 2005; Lindgaard et al., 2009; Montgomery et al., 2000; Reisman, 1996; Tierney, 2001). Classical statistical methods (Multivariate Logistic Regression, Discriminant Analysis, Bayes' Classifiers or kNearest Neighbors), Data Exploration Techniques and Artificial Intelligence, including Neural Networks, Fuzzy Logic, Bayes' Networks, Support Vector Machines, and Classification and Regression Trees are employed in the process of design and implementation (Abdala \& Saeed, 2004; Arif et al., 2010; Bagley et al., 2001; Chen et al., 2009; Forsström \& Dalton, 1995; Huang et al., 2009; Ji et al., 2009; Lisboa \& Taktak, 2006; Long et al., 1993; Pavlopoulos et al., 2004; Polat \& Günes, 2006; Verplancke et al., 2008; Zupan et al., 2001). Among others, these techniques and methods are intended to support the process of performing screening tests, the clinical decision-making process (laboratory procedures are also taken into account), the therapeutic process (including dosage of medications and pharmacoeconomics), and management of the healthcare system in the area of chronic diseases (Berlin et al., 2006; Glaser, 2008; Lenz \& Reuchert, 2007; Tierney, 2001). Estimations show that after implementation, CCDSS generally improved the quality of healthcare in the area of clinical practice by $60-70 \%$ (based on the cases subjected to analysis) and the quality of the management system for chronic diseases by approx. 60\%; moreover, thanks to CCDSS, the quality of pharmaceutical systems improved by over $65 \%$ while patient satisfaction grew remarkably. The essential problem, both in terms of clinical procedures and economics, is assessment of the risk related to adverse event occurrence (such as hospitalization or death). On av- 
erage, the implementation of Clinical Decision Support Systems reduced the risk of misdiagnosis by 16\% (Bairstow et al., 2010; Durieux et al., 2000; Garg et al., 2005; Haynes \& Wilczyński, 2010; Kawamoto et al., 2005; Sintchenko et al., 2005; Thursky et al., 2006).

\section{Clinical Decision Support Systems}

In most of the currently existing healthcare systems, for any patient, both clinical decisions and therapeutic processes are highly complex (Lenz \& Reuchert, 2007; Tierney, 2001). The decision-making process presented in Figure 1 is inherently associated with the limitations imposed by the healthcare system, the clinical environment, the available information, patient preferences, medical personnel preferences, and preferences of the person who manages the medical establishment (Lenz \& Reuchert, 2007; Leslie \& Denvir, 2007).

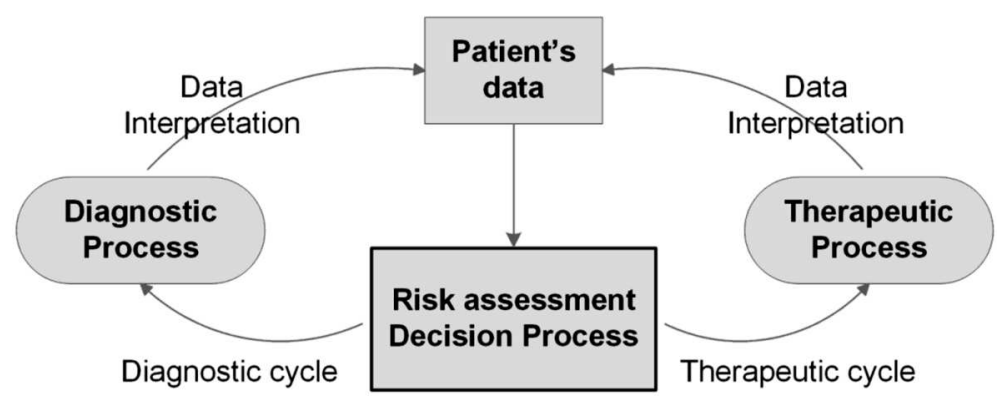

Figure 1. Decision Making Process Diagram (Risk Assessment) covering patient information and both the diagnostic and therapeutic cycles

On the one hand, clinical conditions include the nature, intensity, and complexity of a problem (case) subjected to analysis whereas, on the other hand, they also include the healthcare policy and the economic regime imposed on a clinical medicine specialist by a given institution. Patient preferences are perceived as the most important in the situation when further proceedings are not clearly defined in the decision-making process (there are many ways to make decisions about diagnostic methods and medical treatment). Under such circumstances, patient satisfaction and the quality of their life after treatment must be taken into account. Finally, the decision must also take into account the social and financial limitations of a patient and consider the economics of diagnostic and therapeutic proce- 
dures (Bates et al., 2006; Berner, 2009; Dolan, 2008; Hardy \& Smith, 2008; Lin et al., 2009; Wennberg, 1988). The optimal clinical decision process is carried out according to the following order of actions:

- Establishment of diagnosis,

- Selection of the optimal therapy respective of the current guidelines, instructions, and data outsourced from professional publications (the principles of Evidence Based Medicine),

- Correct application of medical treatment (observance of guidelines, meeting deadlines, and medication dosage),

- Monitoring of therapeutic effects, including side effects,

- Modification of treatment, if any.

A very important aspect of the healthcare system is the possibility of making an incorrect decision. Incorrect decisions result from deficiency of information on a particular patient (situation) at a specific moment, in a specific environment, and can result from uncertainty related to the available data (measurement errors, false positive or false negative results, etc.). In order to reduce the risk of occurrence of an incorrect decision to a minimum, a new paradigm of healthcare is proposed in professional publications and in medical management systems. According to it, formation of multidisciplinary teams of clinical medicine specialists, medical personnel and biostatisticians, and/or biomedical engineers is proposed. The main purpose of actions taken by such teams is to optimize the decision-making process, taking into account not only the clinical environment but also patient satisfaction and the quality of their life. The expected effect of the team's work is an improvement in the functioning of the healthcare system and in the long-term prognosis of the patient's treatment. These are yielded by the developed patterns of proceedings and CCDSS (Bairstow et al., 2010; Kawamoto et al., 2005; Tierney, 2001). The fact that many healthcare systems have already developed effective emergency management methods (acute services), yet can hardly keep up in cases of chronic conditions, is also of certain importance (Young et al., 2007).

\section{Definition, Structure and Examples of CCDSS Applications}

CCDSS systems are variously defined in professional publications. According to Musen (1997), they constitute any fragment of software that enables an analysis of information pertaining to current clinical situations and presents conclusions (guidelines) for a clinician as output information. Berner (1999) defines such systems as a computer algorithm collaborating 
with a clinician in the clinical decision-making process at selected phases of inference (in practice, such systems are commonly known as expert systems). The input data includes clinical symptoms, results of laboratory tests, and image-derived data. The output data constitutes diagnostic and therapeutic guidelines. A CCDSS database contains collected and well-structured knowledge dealing with particular clinical problems (the data is outsourced from experts), while the inference mechanism is a computer algorithm processing input data, taking into account database information. How knowledge is represented and processed determines both the structure and utility of CCDSS (Guilan et al., 2008).

Typical uses of CCDSS include (Berlin et al., 2006; Birkmeyer et al., 2001; Lenz \& Reuchert, 2007; Levy \& Linker, 2008):

- Improvement of the quality of processes of medical data collection through minimization of data deficiencies and reduction of time complexity, which contribute to a more efficient decision-making process,

- Improvement in the monitoring of therapeutic processes and patients' current health condition through presentation of data in a clear and consistent manner,

- Provisions of specific pieces of information required to assess patients' health condition on a day-to-day basis or through generation of alert messages when the assumed value limits or parameters under observation are exceeded (for example, at Intensive Care Units),

- Analysis of discrepancies between the current therapeutic processes and guidelines, as well as instructions, including contraindications and interactions between particular medications,

- Supervision over the planned actions and reminding the medical personnel of the necessity of their implementation,

- Assessment of the risk of disease development and pharmacoeconomic analysis.

Depending on the model, the method of knowledge representation and the inference mechanism employed, various types of CCDSS exist.

Logistic Regression and Discriminant Analysis are very popular statistical methods, employed extensively in medical research. Logistic Regression is a statistical model that enables an analysis of the impact of many variables (both quantitative and qualitative) on a single dichotomic dependent variable (for example, the event of hospitalization or death). Moreover, from the perspective of a clinician, such a model helps to estimate the probability of occurrence of an event defined by a dependent variable (in other words, for example, the risk of death), while the Discriminant Analysis method makes it possible to make decisions in which variables included in the pro- 


\section{Aleksander J. Owczarek et al.}

cess of classification of a group of cases (patients) into predefined subgroups are important (Bagley et al., 2001; Rausch \& Kelley, 2009). In one research project (Wang et al., 2004), the Logistic Regression method was used for the clinical decision process concerning SARS cases and Patient Assessment System was developed, based on the results of laboratory tests and clinical symptoms (12 points in total). The model was compiled based on an analysis of 175 patients suffering from SARS (diagnosed) and then tested on 232 other patients (60 evidenced cases of SARS and 172 control persons). With a defined cutoff value of 6 points, a detection sensitivity of $100 \%$ and a detection specificity of $93 \%$ were achieved. Logistic Regression was also used in the analysis of 30-day survival outcome in a group of 2,500 patients suffering from heart failure. The area under ROC curve (0.734) was the largest in comparison with Neural Network and k-Nearest Neighbors algorithms (Philips \& Street, 2005). Similarly, based on an analysis of a group consisting of 672 patients suffering from chest pain, an assessment of the risk of coronary heart disease was developed and the following results were achieved on a validation group (774 patients): a detection sensitivity of $86 \%$ and a detection specificity of $47 \%$ (Gencer et al., 2010). The Discriminant Analysis method was also employed to determine mortality outcome factors in a group of 67 patients suffering from idiopathic cardiomyopathy (Martí et al., 1997). The achieved quality of classification was $82 \%$. Moreover, Discriminant Analysis as well as (multinomial) Logistic Regression was used in a group of 3,566 patients hospitalized after cardiac infarction in order to determine the variables that enable an estimation of the time between the moment at which cardiac infarction symptoms appeared and the moment of arrival at a hospital (Hossain et al., 2002). The average quality of classification was $62.4 \%$.

The use of Artificial Intelligence (AI) for CCDSS includes, among others, Artificial Neural Networks, Fuzzy Logic, Neuro-Fuzzy Systems, Bayes' and Semantic Networks, Classification and Decision Trees, and Support Vector Machines (SVM), or various types of data clustering methods. Artificial Neural Networks (ANN), one of the most potent branches of AI, are mathematical models performing calculations by means of elements known as artificial neurons, connected with each other by synapses, representing contact weights (acquired in the process of learning and reflecting the acquired knowledge) (Ajith, 2005). An example application of ANN in 1-year survival outcome of 96 patients suffering from heart failure, based on clinical and echocardiographic data, enabled the development of a prediction model, with a classification quality of $90 \%$, a sensitivity of $93 \%$, and a specificity of $71 \%$ (Ortiz et al., 1995). In another project, the au- 
thors presented an application of the algorithm for k-means clustering and neural networks in order to assess the risk of occurrence of acute coronary syndrome and managed to develop appropriate decision rules (Shantakumar \& Kumaraswamy, 2009). Neural networks were also used to predict the occurrence of brain stroke; the achieved qualification was $98 \%$ (Shanti et al., 2009). A review of different structures and various types of neural networks combined with data dimensionality reduction based on principal component analysis, employed in cardiovascular disease prediction, is presented by Raut and Dudul (2010). For the best network, i.e. multilayer perceptron, an average classification quality of $97 \%$ was achieved.

Support Vector Machine (SVM) is a classifier determining the process of learning the optimal hyperplane, separating data belonging to two opposite (various) classes, with possibly the largest margin of confidence. There are several types of support vectors with various base functions: linear, polynomial, radial, and sigmoidal. In one paper, ECG spectrum analysis was presented (Jankowski et al., 2007), performed in a group of 299 patients suffering from ventricular tachycardia and in a group of 77 healthy volunteers. Using an appropriate signal filtration and an SVM classifier, a classification quality of $95 \%$ was achieved, while Heart Rate Variability analysis with the use of SVM, based on five features selected out of 15 (with the Discriminant Analysis method), makes it possible to achieve a mean quality of classification of $99 \%$ for 5 various arrhythmias (Asi et al., 2008). SVMbased CCDSS was also used to detect heart ventricle contractility abnormality, based on Doppler echocardiography signal analysis, with the achieved values of sensitivity and specificity of $94.5 \%$ and $90 \%$, respectively (Comak et al., 2007).

Other example applications include: the use of Ontology and Semantic Networks (Colantonio et al., 2007; Ragab et al., 2004), Analysis of Principal Components, Regression (Jilani et al., 2013), Fuzzy Logics (Setiawan et al., 2009), Possibility Approach, Bayes' Networks, and Classification Trees that enable the representation of uncertain knowledge in the form of an acyclic digraph (nodes representing variables and arches representing inter-node contacts) (Adams et al., 2008; Atoui et al., 2006; Fonarow, 2008; Fonarow et al., 2005; Kurt et al., 2008; Mahesh et al., 2009). A sample prediction model, with the use of Classification and Regress Tree (CART), for the assessment of the risk of death in a hospital for patients suffering from decompensate heart failure was presented by Fonarow et al. (2005). The model was developed based on the results from a group of 33,046 patients and verified on a group of 32,229 patients. 


\section{The Effects of CCDSS Use in Clinical Practice}

CCDSS provide medical personnel with appropriate and precisely defined pieces of timely information; hence, they enable an improvement in the quality of healthcare and medical services as well as an increase in patient satisfaction. Furthermore, they reduce healthcare system costs (Kawamoto et al., 2005). Kawamoto et al. (2005) presented a systematic review of 88 professional publications related to CCDSS. 71 systems were evaluated by 6,000 clinical medicine specialists. The group of patients was composed of about 130,000 individuals. The implementation of 48 systems out of those 71 (68\%; 95\% CI: 56-78\%) remarkably improved the quality of clinical practice. Moreover, an analysis of meta-regression led to the conclusion that, when a Clinical Decision Support System is being designed, the following 4 features are important in statistical terms:

- Automatic support provided to a clinician in the decision-making process $(\mathrm{OR}=112.1 ; \mathrm{p}<0.001)$,

- Support provided at the right place and at the right time $(\mathrm{OR}=15.4$; $\mathrm{p}<0.05)$,

- Provision of specific guidelines $(\mathrm{OR}=7.1 ; \mathrm{p}<0.05)$,

- Implementation of a computer-based system $(\mathrm{OR}=6.3 ; \mathrm{p}<0.05)$.

Of the 32 analyzed systems satisfying the above requirements, 30 (94\%; 95\% CI: 80-99\%) were found to improve the quality of clinical practice to a huge extent. A systematic review of professional papers, covering the appraisal of the impact of CCDSS on the quality of healthcare and the long-term prognosis of treatment, was presented by Garg et al. (2005). Of the 97 systems subjected to analysis, 62 (64\%; 95\% CI: 54-73\%) contributed to remarkable improvement of healthcare quality. This group included 10 clinical decision support systems, the scope of which covered decisions taken in the following areas: psychological disorders (remarkable improvement observed) and psychiatric disorders (including depression), Acute Coronary Syndromes (remarkable improvement observed), chest pain, ECG in Coronary Syndromes (remarkable improvement observed), Acute Abdominal Cavity Pain and gastroenterology (remarkable improvement observed). Aggregately, remarkable improvement of healthcare quality was achieved in 4 cases (40\%; 95\% CI: 17-69\%). 37 systems were used for the management of chronic diseases and preventive actions, including: diabetes (screening tests included: remarkable improvement) and cardiovascular diseases (including arterial blood pressure risk assessment: remarkable improvement, risk of the occurrence of adverse heart events: remarkable improvement, pharmacotherapy, and antiplatelet treatment). Re- 
markable improvement of healthcare quality was observed in 23 systems (62\%; 95\% CI: $46-76 \%)$.

The frequency of misdiagnosis in cases of imaging were subjected to analysis in a group of 423 patients examined by medical practitioners in the following conditions: suspicion of pulmonary thromboembolism, diagnostics of bonelet injury, diagnostics of nephritic colic, and acute abdominal pain not caused by trauma (Bairstow et al., 2010). Indeed, statistically, the implementation of CCDSS remarkably reduced the number of incorrect diagnostic and therapeutic actions, i.e. by $13 \%, 28 \%, 22 \%$, and $17 \%$, respectively; on average, the reduction was $16 \%$ (95\% CI: $13-20 \%$; p < 0.001) (Levy \& Linker, 2008). In summary, in the case of ca. $67 \%$ (95\% CI: 62 $71 \%$ ) of CCDSS, the use of the systems in question results in remarkable improvement of healthcare quality, including the reduced risk of an improper clinical decision (Birkmeyer et al., 2001).

\section{Conclusions}

In order to minimize the risk of improper clinical decision making and help guarantee proper analyses, correct interpretation of results, and verification of research hypotheses, the creation of multidisciplinary teams of clinicians, medical personnel, biostatisticians and/or biomedical engineers seems to be a sine qua non. Precisely and accurately designed and implemented Computerized Medical Decision Support Systems enable the improvement of healthcare quality and medical service; they also increase patient satisfaction and reduce the burden on healthcare system.

\section{R E F E R E N C E S}

Abdala, O., \& Saeed, M. (2004). Estimation of Missing Values in Clinical Laboratory Measurements of ICU Patients Using a Weighted K-Nearest Neighbors Algorithm. Computers in Cardiology, 31, 693-696.

Adams, K., Uddin, N., \& Patterson, J. (2008). Clinical predictors of in-hospital mortality in acutely decompensated heart failure-piecing together the outcome puzzle. Congestive Heart Failure, 14(3), 127-134.

Ajith, A. (2005). Artificial Neural Networks. In P. H. Sydenham \& R. Thorn (Eds.), Handbook for Measurement Systems Design (pp. 901-908). London: John Wiley and Sons Ltd.

Arif, M., Akram, M., \& Minhas, F. (2010). Pruned fuzzy K-nearest neighbor classifier for beat classification. Journal of Biomedical Science and Engineering, $3(4), 380-389$. 
Asi, B., Setarehdan, S., \& Mohebbi, M. (2008). Support vector machine-based arrhythmia classification using reduced features of heart rate variability signal. Artificial Intelligence in Medicine, 44(1), 51-64.

Atoui, H., Fayn, J., Gueyffier, F., \& Rubel, P. (2006). Cardiovascular Risk Stratification in Decision Support Systems: A Probabilistic Approach. Application to Health. Computers in Cardiology, 33, 218-284.

Bagley, S., White, H., \& Golomb, B. (2001). Logistic regression in the medical literature: Standards for use and reporting, with particular attention to one medical domain. Journal of Clinical Epidemiology, 54(10), 979-985.

Bairstow, P., Persaud, J., Mendelson, R., \& Ngyuen, L. (2010). Reducing inappropriate diagnostic practice through education and decision support. International Journal for Quality in Health Care, 22(3), 194-200.

Bates, D., Kuperman, G., Wang, S., Gandhi, T., Kittler, A., Volk, L., Spurr, C., et al. (2006). Ten commandments for effective clinical decision support: making the practice of evidence-based medicine a reality. Journal of the American Medical Informatics Association, 10(6), 523-530.

Berlin, A., Sorani, M., \& Sim, I. (2006). A taxonomic description of computerbased clinical decision support systems. Journal of Biomedical Informatics, 39(6), 656-667.

Berner, E. S. (Ed.). (1999). Clinical decision support systems: theory and practice (pp. 3-30). Germany: Springer.

Berner, E. S. (Ed.). (2009). Clinical Decision Support Systems: State of the Art (AHRQ Publication No. 09-0069-EF). Rockville, Maryland: Agency for Healthcare Research and Quality.

Birkmeyer, J., Schwartz, L., Sargent, J., \& Woloshin, S. (2001). Computer-Based Decision Support. Wishing on a Star? Effective Clinical Practice, 4(1), 34-38.

Chen, S., Hsiao, Y., Huang, Y., Kupo, S., Tseng, H., Wu, H., \& Chen, D. (2009). Comparative Analysis of Logistic Regression, Support Vector Machine and Artificial Neural Network for the Differential Diagnosis of Benign and Malignant Solid Breast Tumors by the Use of Three-Dimensional Power Doppler Imaging. Korean Journal of Radiology, 10(5), 464-471.

Colantonio, S., Martinelli, M., Moroni, D., Salvetti, O., Perticone, F., Sciacqua, A., Conforti, D., \& Gualtieri, A. (2007). An approach to decision support in heart failure. CEUR Workshop Proceedings, 314, 1-10.

Comak, E., Arslan, A., \& Türkoglu, I. (2007). A decision support system based on support vector machines for diagnosis of the heart valve diseases. Computers in Biology and Medicine, 37(1), 21-27.

Dobbson, A. (1983). The role of Statistician. International Journal of Epidemiology, 12(3), 274-275.

Dolan, J. (2008). Shared decision-making - transferring research into practice: the Analytic Hierarchy Process (AHP). Patient Education and Counseling, 73(3), 418-425. 
Durieux, P., Nizard, R., Ravaud, P., Mounier, N., \& Lepage, E. (2000). A clinical decision support system for prevention of venous thromboembolism: effect on physician behavior. JAMA, 283(21), 2816-2821.

Eom, J., Kim, S., \& Zhang, B. (2008). AptaCDSS-E: A classifier ensemble-based clinical decision support system for cardiovascular disease level prediction. Expert Systems with Applications, 34(4), 2465-2479.

Fonarow, G. (2008). Epidemiology and risk stratification in acute heart failure. American Heart Journal, 155(2), 200-207.

Fonarow, G., Adams, K., Abraham, W., Yancy, C., \& Boscardin, W. (2005). Risk Stratification for In-Hospital Mortality in Acutely Decompensated Heart Failure - classification and regression tree analysis. JAMA, 293(5), 572-580.

Forsström, J., \& Dalton, K. (1995). Artificial neural networks for decision support in clinical medicine. Annals of Medicine, 27(5), 509-517.

Garg, A., Adhikari, N., McDonald, H., Rosas-Arellano, M., Devereaux, P., Beyene, J., \& Haynes, R. (2005). Effects of computerized clinical decision support systems on practitioner performance and patient outcomes. A Systematic Review. JAMA, 293(10), 1223-1238.

Gencer, B., Vaucher, P., Herzig, L., Verdon, F., Ruffieux, C., Bösner, S., \& Favrat, B. (2010). Rulling out coronary heart disease in primary care patients with chest pain: a clinical prediction score. BMC Medicine, 8(9), 1-10.

Glaser, J. (2008). Clinical decision support: the power behind the electronic health record. Healthcare Financial Management, 62(7), 50-51.

Guilan, K., Dong-Ling, X., \& Jian-Bo, Y. (2008). Clinical decision support systems: a review on knowledge representation and inference under uncertainties. International Journal of Computational Intelligence Systems, 1(2), 159-167.

Hardy, D., \& Smith, D. (2008). Decision making in clinical practice. British Journal of Anaesthetic \& Recovery Nursing, 9(1), 19-21.

Haynes, R., \& Wilczyński, N. (2010). Effects of computerized clinical decision support systems on practitioner performance and patient outcomes: Methods of a decision maker-research partnership systemic review. Implementation Science, 5:12.

Hossain, M., Wright, S., \& Pertersen, L. (2002). Comparing performance of multinomial logistic regression and discriminant analysis for monitoring access to care for acute myocardial infarction. Journal of Clinical Epidemiology, 55(4), 400-406.

Huang, D., Quan, Y., He, M., \& Zhou, B. (2009). Comparison of linear discriminant analysis methods for the classification of cancer based on gene expression data. Journal of Experimental \& Clinical Cancer Research, 28(1), 149-156.

Hughes, M. C. (2009). Using clinical decision support to improve health and achieve cost savings (Anvita Health Report). Retrieved from http://anvitahealth. com/... pdf/AnvitaHealth20Report-CDSROI.pdf 
Jankowski, S., Szymański, Z., Piątkowska-Janko, E., \& Oreziak, A. (2007). Improved recognition of sustained ventricular tachycardia from SAECG by support vector machine. The Anatolian Journal of Cardiology, r(Suppl 1), $112-115$.

Ji, S., Smith, R., Huynh, T., \& Najarian, K. (2009). A comparative analysis of multi-level computer-assisted decision making systems for traumatic injuries. BMC Medical Informatics and Decision Making, 9:2, 2-18.

Jilani, T., Yasin, H., Yasin, M., \& Ardil, C. (2013). Acute coronary syndrome prediction using data mining techniques - an application. World Academy of Science, Engineering and Technology, International Journal of Computer and Information Engineering, 7(1), 168-172.

Kawamoto, K., Houlihan, C., Balas, E., \& Lobach, D. (2005). Improving clinical practice using clinical decision support systems: a systematic review of trials to identify features critical to success. BMJ, 330(7494), 765-772.

Kurt, I., Ture, M., \& Kurum, A. (2008). Comparing performances of logistic regression, classification and regression tree, and neural networks for predicting coronary artery disease. Expert Systems with Applications, 34(1), 366-374.

Lenz, R., \& Reuchert, M. (2007). IT support for healthcare process - premises, challenges, perspectives. Data \& Knowledge Engineering, 61, 39-58.

Leslie, L., \& Denvir, M. (2007). Clinical decision support software for chronic heart failure. Critical Pathways in Cardiology: A Journal of Evidence-Based Medicine, 6(3), 121-126.

Levy, W., \& Linker, D. (2008). Prediction of Mortality in Patients with Heart Failure and Systolic Dysfunction. Current Cardiology Report, 10(3), 198205.

Lin, C., Lin, C., Lin, B., \& Yang, M. (2009). A decision support system for improving doctor's prescribing behavior. Expert Systems with Applications, 36(4), 7975-7984.

Lindgaard, G., Pyper, C., Frize, M., \& Walker, R. (2009). Does Bayes have it? Decision Support Systems in diagnostic medicine. International Journal of Industrial Ergonomics, 39(3), 524-532.

Lisboa, P., \& Taktak, A. (2006). The use of artificial neural networks in decision support in cancer: A systematic review. Neural Networks, 19(4), 408-415.

Long, W., Griffith, L., Selker, H., \& D'Agostino, R. (1993). A comparison of logistic regression to decision-tree induction in a medical domain. Computers in Biomedical Research, 26(1), 74-97.

Mahesh, V., Kandaswamy, A., Vimal, C., \& Sathish, B. (2009). ECG arrhythmia classification based on logistic model tree. Journal of Biomedical Science and Engineering, 2(6), 405-411.

Martí, V., Ballester, M., Marrugat, J., Auge, J., Padro, J., Narula, J., \& Caralps, J. (1997). Assessment of the appropriateness of the decision of heart transplantation in idiopathic-dilated cardiomyopathy. The American Journal of Cardiology, 80(6), 746-750. 
Montgomery, A., Fahey, T., Peters, T., MacIntosh, C., \& Sharp, D. (2000). Evaluation of computer based clinical decision support system and risk chart for management of hypertension in primary care: randomised controlled trial. BMJ, 320(7236), 686-690.

Musen, M. A. (1997). Methods for decision support. In M. A. Musen \& J. H. van Bemel (Eds.), Handbook of medical informatics (pp. 233-246). Germany: Springer.

Ortiz, J., Ghefter, C., Silva, C., \& Sabbatini, R. (1995). One-year mortality prognosis in heart failure: A neural network approach based on echocardiographic data. Journal of the American College of Cardiology, 26(7), 1586-1593.

Pavlopoulos, S., Stasis, A., \& Loukis, E. (2004). A decision tree-based method for the differential diagnosis of Aortic Stenosis from Mitral Regurgitation using heart sounds. BioMedical Engineering Online, 3:21, 21-35.

Philips, K., \& Street, W. (2005). Predicting outcomes of hospitalization for heart failure using logistic regression and knowledge discovery methods. In $A M I A$ 2005 Annual Symposium Proceedings (pp. 1080).

Polat, K., \& Günes, S. (2006). A hybrid medical decision making system based on principles component analysis, k-NN based weighted pre-processing and adaptive neuro fuzzy inference system. Digital Signal Processing, 16(6), 913921.

Ragab, A., Fakeeh, K., \& Roushdy, M. (2004). A medical multimedia expert system for heart diseases diagnosis and treatment. In Proceedings of the 2nd Saudi Science Conference (pp. 31-45). Jeddah, Kingdom of Saudi Arabia.

Rausch, J., \& Kelley, K. (2009). A comparison of linear and mixture models for discriminant analysis under abnormality. Behavior Research Methods, 41 1 (1), $85-98$.

Raut, R., \& Dudul, S. (2010). Intelligent diagnosis of heart diseases using neural network approach. International Journal of Computer Applications, 1(2), $117-123$.

Reisman, Y. (1996). Computer-based clinical decision aids. A review of methods and assessment of systems. Medical Informatics, 21(3), 179-197.

Setiawan, N., Venkatachalam, P., \& Hani, A. (2009). Diagnosis of coronary artery disease using artificial intelligence based decision support system. In Proceedings of the International Conference on Man-Machine Systems (pp. 1C3-11C3-5). Batu Ferringhi, Penang, Malaysia

Shantakumar, B., \& Kumaraswamy, Y. (2009). Intelligent and effective heart attack prediction system using data mining and artificial neural networks. European Journal of Scientific Research, 31(4), 642-656.

Shanti, D., Sahoo, G., \& Saravanan, N. (2009). Designing an artificial neural network model for the prediction of thrombo-embolic stroke. International Journal of Biometrics and Bioinformatics, 3(1), 10-18. 
Sim, I., Gorman, P., Greenes, R., Haynes, R., Kaplan, B., Lehmann, H., \& Tang, P. C. (2001). Clinical decision support systems for the practice of evidencebased medicine. Journal of the American Medical Informatics Association, $8(6), 527-534$.

Sintchenko, V., Iredell, J., Gilbert, G., \& Coiera, E. (2005). Handheld computerbased decision support reduces patient length of stay and antibiotic prescribing in critical care. Journal of the American Medical Informatics Association, 12(4), 398-402.

Szydło, R. (2005). Komu jest potrzebny statystyk medyczny? Onkologia w Praktyce Klinicznej, 1(3), 129-131.

Thursky, K., Buising, K., Bak, N., Macgregor, L., Street, A., Macintyre, C., Brown, G., et al. (2006). Reduction of broad-spectrum antibiotic use with computerized decision support in an intensive care unit. International Journal for Quality in Health Care, 18(3), 224-231.

Tierney, W. (2001). Improving clinical decision and outcomes with information: a review. International Journal of Medical Informatics, 62(1), 1-9.

Verplancke, T., Van Looy, S., Benoit, D., Vansteelandt, S., Depuydt, P., De Turck, F., \& Decruyenaere, J. (2008). Support vector machine versus logistic regression modeling for prediction of hospital mortality in critically ill patients with haematological malignancies. BMC Medical Informatics and Decision Making, 8:56.

Wang, T., Jang, T., Huang, C., Kao, S., Lin, C., Lee, F., Liu, C., et al. (2004). Establishing a clinical decision rule of severe acute respiratory syndrome at the emergency department. Annals of Emergency Medicie, 43(1), 17-22.

Wennberg, J. (1988). Improving the medical decision-making process. Health Affairs, $7(1), 99-106$.

Young, A. S., Chaney, E., Shoai, R., Bonner, L., Cohen, A. N., Doebbeling, B., Dorr, D., et al. (2007). Information technology to support improved care for chronic illness. Journal of General Internal Medicine, 22(Suppl. 3), 425-430.

Zupan, B., Porenta, A., Vidmar, G., Aoki, N., Bratko, I., \& Beck, J. (2001). Decision at hand: a decision support system on handhelds. Studies in Health Technology and Informatics, 84(1), 566-570. 